\title{
AIDS Malignancy Consortium
}

National Cancer Institute

\section{Source}

National Cancer Institute. AIDS Malignancy Consortium. NCI Thesaurus. Code C19707.

Established in 1995 to carry out Phase I and II studies of innovative therapies for AIDS malignancies. 